\title{
Diagnosis of Submucosal Tumors by Injecting a Water Soluble Contrast Medium : Diagnosis of Extra-Gastric Tumors and Gastric Varices
}

\author{
Shigeru Asaki, Yo Shishido, Setsuko Miyoshi, Waichi \\ Sato, Hiroshi Sato, Tadashi Motozima and Shinya \\ Meguro
}

The Third Department of Internal Medicine, Tohoku University School of Medicine, Sendai 980

\begin{abstract}
Asaki, S., Shishido, Y., Miyoshi, S., Sato, W., Sato, H., Мotozima, T. and Meguro, S. Diagnosis of Submucosal Tumors by Injecting a Water Soluble Contrast Medium: Diagnosis of Extra-Gastric Tumors and Gastric Varices Tohoku J. exp. Med., 147 (3), $235-245 —$ Extra-gastric compression caused tumescent lesions that are difficult to differentiate from gastric submucosal tumors, and gastric varices similar in appearance to circumscribed tumors were sometimes experienced clinically. Up to now, the differential diagnosis of these lesions has been done by the palpation through $\mathrm{x}$-ray examination or the tactile test under endoscopic examination. Even by the recent use of the CT scan, the differential diagnosis still remains unsatisfactory except in a few specific cases. Under these present circumstances, submucosography is recommended for routine screening test for outpatients. Our method is simple, safe and time-saving. Recently, it has become easy to diagnose hemangiomas as well as varices in the stomach by application of submucosography. Accordingly, in the cases of vascular tumors or tumescent lesions caused by extra-gastric compression, the risk of incidental major bleeding or perforation can be prevented by using the submucosography.

submucosography ; submucosal tumor ; gastric varix ; hemangioma ; extra-gastric compression
\end{abstract}

It has sometimes been difficult to differentiate between gastric submucosal tumors and other tumescent lesions caused by extra-gastric pressure. The differentiation has so far been attempted roentgenographically through confirmation of the relation between the tumor and the gastric wall by palpation under fluoroscopy or by checking the changes in the appearance of the tumor from various angles. On the other hand, endoscopical identification of the tumor has been made by confirming, under direct vision, whether the tumor moves in accordance with the gastric wall during breathing, or by confirming the tumor's relation with the gastric wall by tactile test using biopsy forceps. Even by these measures, the discrimination has often been unsuccessful when the tumor is

Received March 5, 1985 ; accepted for publication August 14, 1985. 
located in a position out of the reach of palpation under $\mathrm{x}$-ray examination, as in case of its adhesion to the gastric wall or lying at the gastric fundus. Abdominal angiography (Fujii et al. 1972) has been used in the diagnosis of gastric submucosal tumor and has been proven to be useful, but it is not always easy to delineate the tumor, especially in the case of a small one. The differential diagnosis described in this paper is between submucosal tumors comparatively large in size and confusing tumescent lesions caused by compression of extragastric tumor.

On the contrary, diagnosis of gastric varix is not always easy, especially in case without accompanying esophageal varix. In these cases, careless biopsy somtimes causes critical bleeding, eventually requiring an emergency operation. In the case of a tumor suggesting non-varix or malignancy, it seems necessary to perform gastric biopsy under direct vision. Moreover, an endoscopic tactile test using biopsy forceps and observation of the lesion from different angles appear important as well as for excluding varices to avoid a risk of serious bleeding caused by biopsy. Furthermore, as described in a previous paper (Asaki et al. 1982), our method of contrast x-ray imaging (submucosography) is able to visualize the blood vessels running through the submucosal space, and is considered equally helpful for identifying a varix from its relation to the adjacent blood vessels.

In the present paper, we describe some cases of gastric varix diagnosed by analysis of submucosographic manifestations and other types of gastric varix showing circumscribed tumors.

\section{Materials}

Among a total of 277 cases with gastric protruded type lesions resembling submucosal tumors, experienced in the Third Department of Internal Medicine, Tohoku University Hospital, since April, 1973, 7 cases were confirmed to be caused by pressure of an extragastric mass. Gastric varix was found in 5 cases, while the remaining 2 cases had venous tumescences resembling a circumscribed type tumor. The results of our submucosographic examinations of these cases are described below.

\section{RESULTS}

Diagnosis of tumescent lesions caused by compression by extra-gastric tumors

Application of our submucosography confirmed that 6 of the 7 gastric protruded lesions had been caused by a compression from extra-gastric masses, i. e., pancreatic tumor, splenic cyst and hepatic hemangioma (Table 1). But, one case was not correctly diagnosed by submucosography because of unsuitable injection of contrast medium.

\section{Case 1}

This 70-year-old female patient visited our outpatient clinic with a chief complaint of heart burn. Gastric roentgenography confirmed a mass at the gastric 
TABLE 1. Extra-gastric tumor cases which were examined by submucosography

\begin{tabular}{ccllcl}
\hline $\begin{array}{c}\text { Case } \\
\text { No. }\end{array}$ & Age & Sex & Location & $\begin{array}{c}\text { Submucosographical } \\
\text { diagnosis }\end{array}$ & Final diagnosis \\
\hline 1 & 72 & Male & Corpus & Extraluminal type* & Hepatoma \\
2 & 68 & Male & Corpus & Extraluminal compression & Pancreatic cancer \\
3 & 61 & Female & Fundus & " & Splenic cyst \\
$4 \dagger$ & 70 & Female & Fundus & " & Hepatic hemangioma \\
5 & 30 & Female & Fundus & " & Splenic cyst \\
6 & 75 & Male & Antrum & " & Pancreatic cancer \\
7 & 67 & Female & Antrum & Gallbladder cancer \\
\hline
\end{tabular}

* Insufficient injection of contrast medium.

$\dagger$ Case presented in the paper.

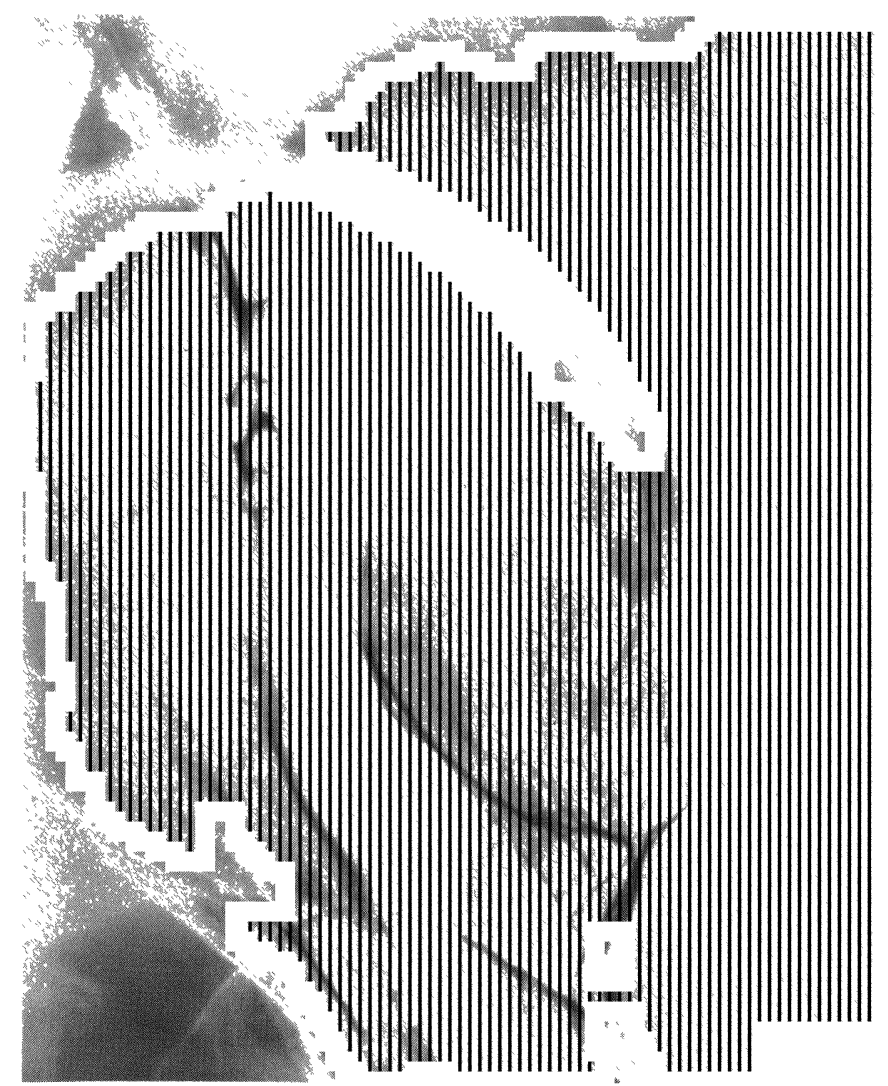

Fig. 1. $x$-Ray picture showing a submucosal tumor-like lesion located in the fundus of the stomach.

fundus. As the surface was covered with a smooth gastric mucosa and the mass was bulging upward gently, a tentative diagnosis was made as a submucosal tumor or a tumescent lesion caused by compression from an extra-gastric mass (Fig. 1). 


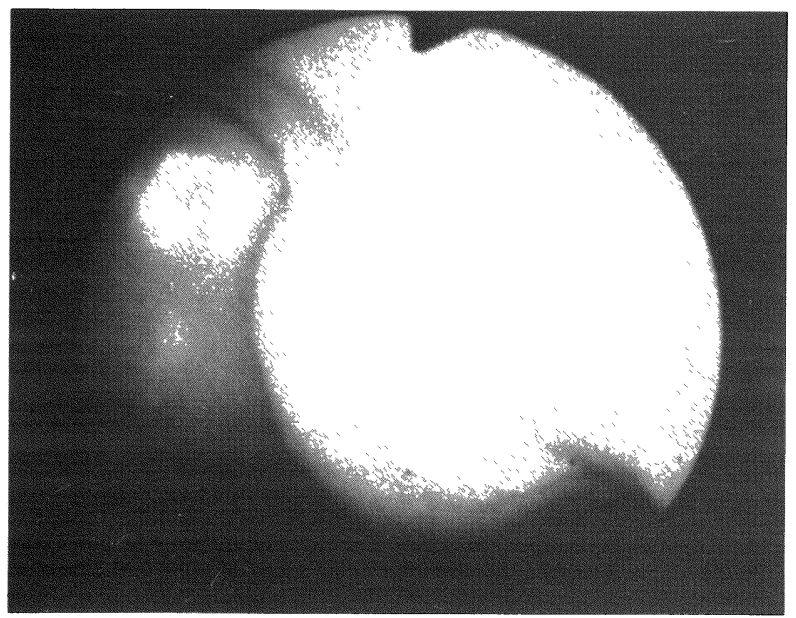

Fig. 2. Endoscopic picture of the tumor-like lesion shown in Fig. 1.

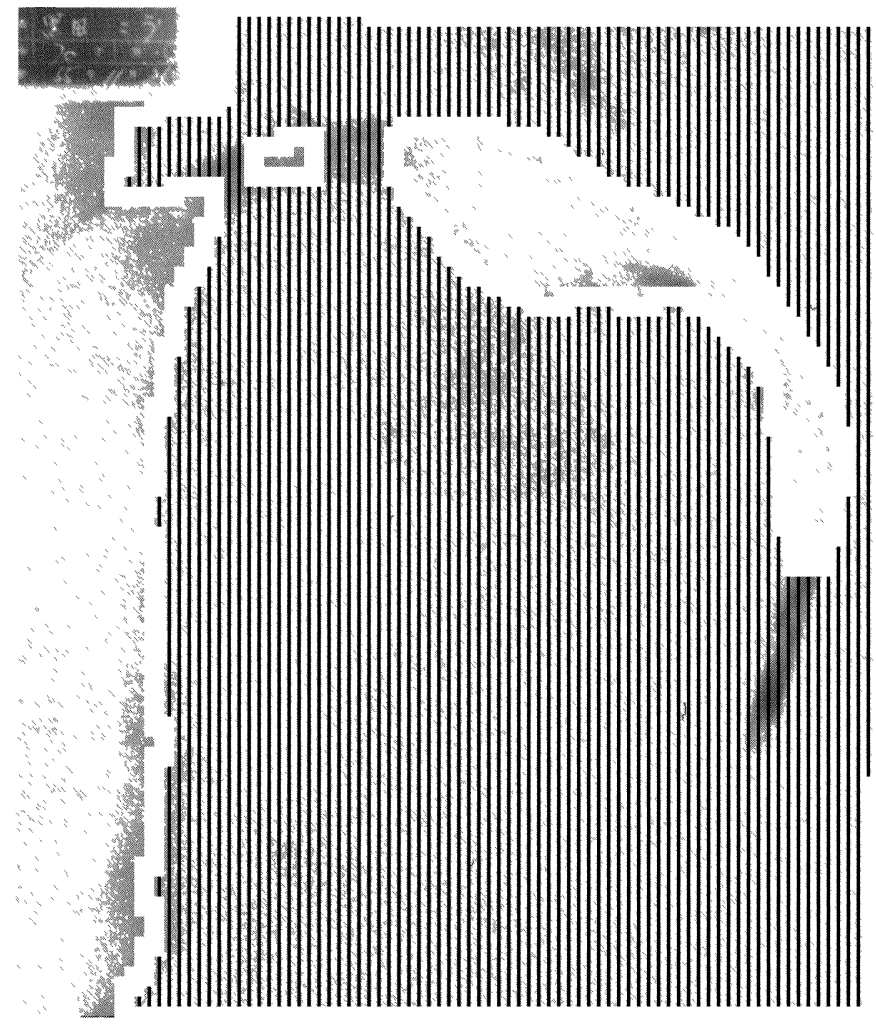

Fig. 3. Submucosography of the tumor-like lesion in Fig. 1. The findings of the separation of contrast medium from the tumor shadow indicated the mass as a tumescent lesion induced by compression of an extra-gastric tumor. 
Endoscopically, the mass with bridging folds on the anterior wall of the gastric fundus had a normal gastric mucosa over the surface, strongly suggesting a gastric submucosal tumor (Fig. 2). Submucosography was applied to this mass. It confirmed an obvious separation of the contrast medium from the tumor shadow, indicating that the tumescent lesion was induced by compression of an extragastric mass in the left hepatic lobe (Fig. 3). The patient was hospitalized and received abdominal CT scan, ultrasonic examination, abdominal angiography, and peritoneoscopy. As a result, she was diagnosed as having a hepatic hemangioma, and underwent surgery. The tumor was finally confirmed to be a cavernous hemangioma.

\section{Diagnosis of gastric varix and gastric hemangioma}

The submucosographic analysis was able to identify gastric varices without esophageal varix in 3 cases, and in two cases of varix resembling gastric submucosal tumors, in total, in 5 cases of gastric venous tumescences. There was another instance of cavernous hemangioma, an example of vascular tumor, that could be diagnosed preoperatively by the submucosographic tracing of the relation between a mass and its surrounding blood vessels (Table 2).

\section{Case 2}

This 36-year-old female patient visited our outpatient clinic with a chief complaint of abdominal fullness. A circumscribed mass irregular in shape at the gastric fundus was shown by the $\mathrm{x}$-ray examination (Fig. 4). The mass was suggestive of a gastric cancer, submucosal tumor, or an extragastric compression. The endoscopic picture suggested a submucosal tumor (Fig. 5). The x-ray picture by submucosography in the frontal view clearly demonstrated the mass was a gastric varix (Fig. 6).

\section{Case 3}

This 69 -year-old male patient was initially received an $\mathrm{x}$-ray examination in

TABLE 2. Gastric varix or hemangioma cases which were diagnosed as vascular tumor by submucosography

\begin{tabular}{ccllc}
\hline Case No. & Age & Sex & Location & Final diagnosis \\
\hline 1 & 23 & Female & Fundus & Venous tumescence \\
$2^{*}$ & 69 & Male & Fundus & " \\
3 & 62 & Female & Fundus & Gastric varix \\
4 & 63 & Male & Fundus & " \\
5 & 53 & Male & Fundus & " \\
$6^{*}$ & 36 & Female & Fundus & " \\
7 & 53 & Female & Fundus & " \\
\hline
\end{tabular}

* Cases presented in this paper. 


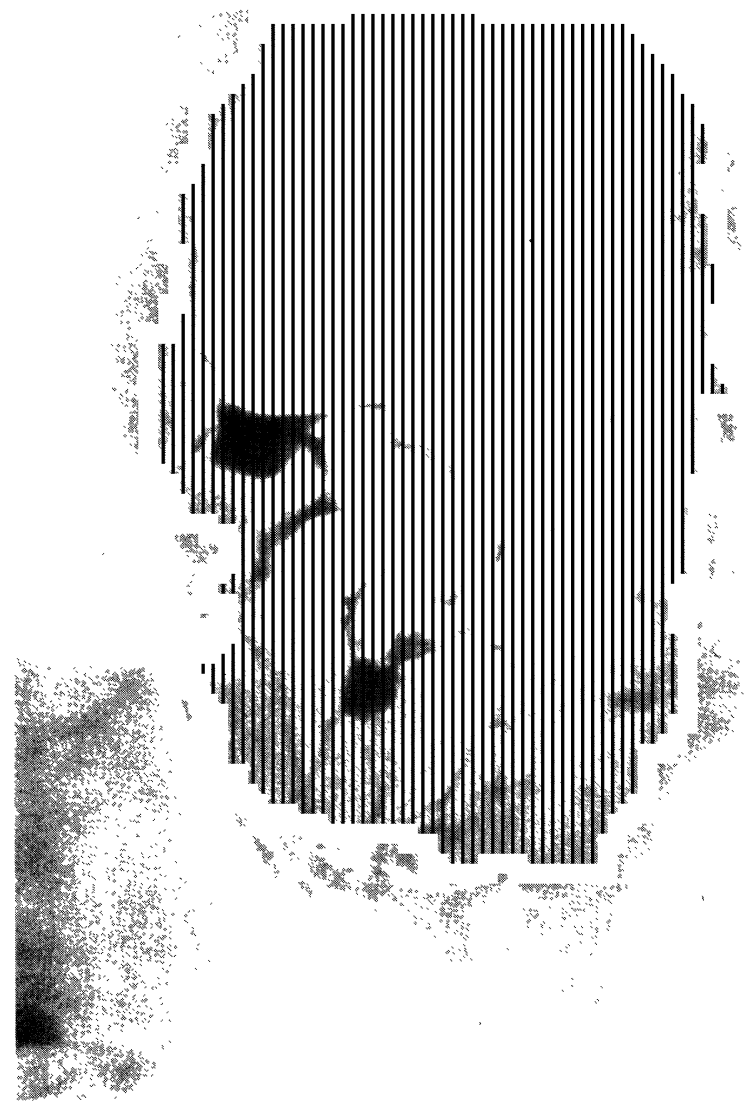

Fig. 4. X-ray picture showing a circumscribed mass irregular in shape at the gastric fundus.

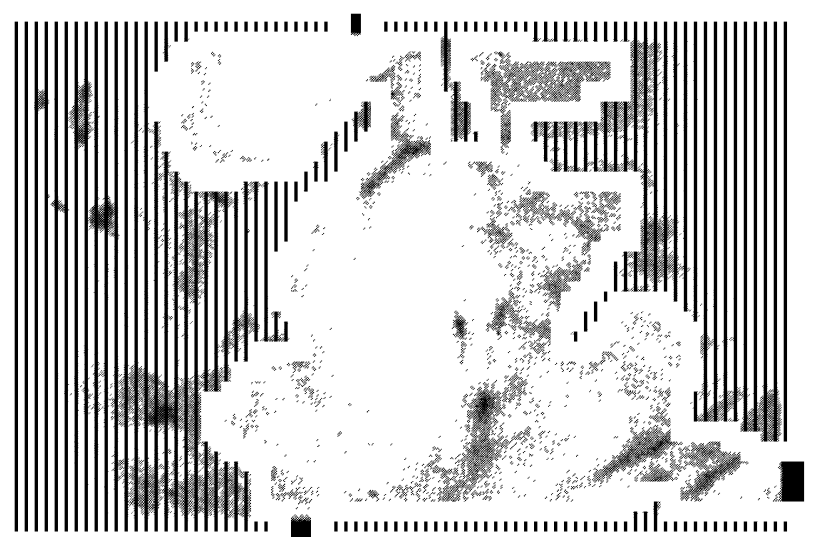

Fig. 5. Endoscopic picture of the mass shown in Fig. 4. 


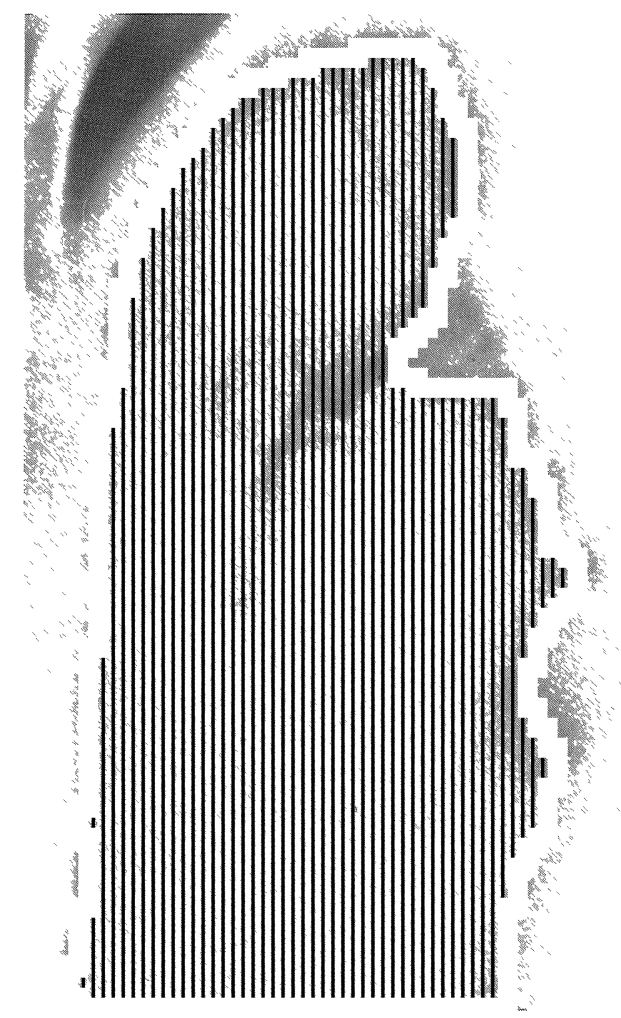

Fig. 6. Submucosography of the mass shown in Fig. 4. The findings of the frontal view of submucosography revealed the tumor as a gastric varix from the relation of the mass with the surrounding several veins.

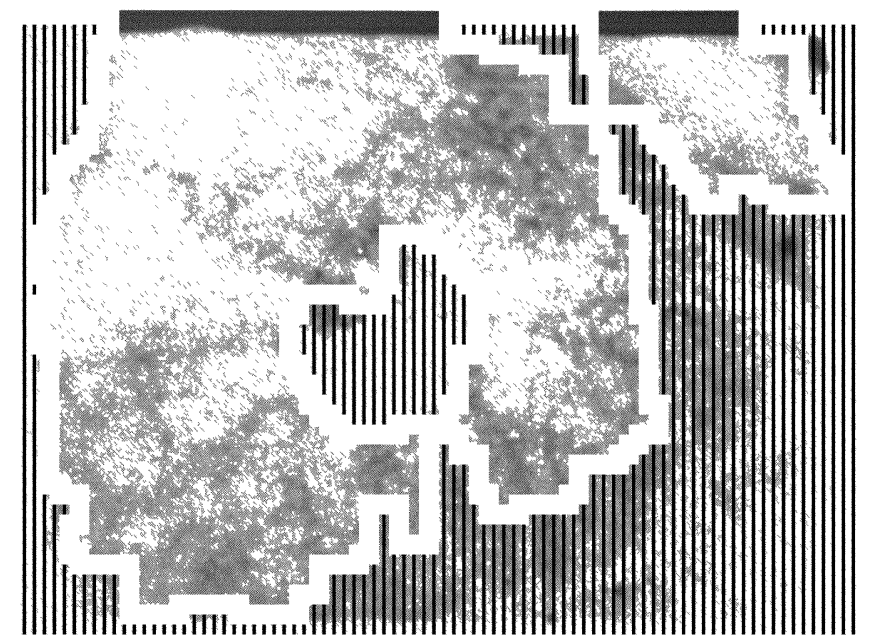

Fig 7. Endoscopic picture taken by gastrocamera GTF type $S_{3}$ (made by Olympus Co.) showing a cavernous hemangioma of the stomach. 


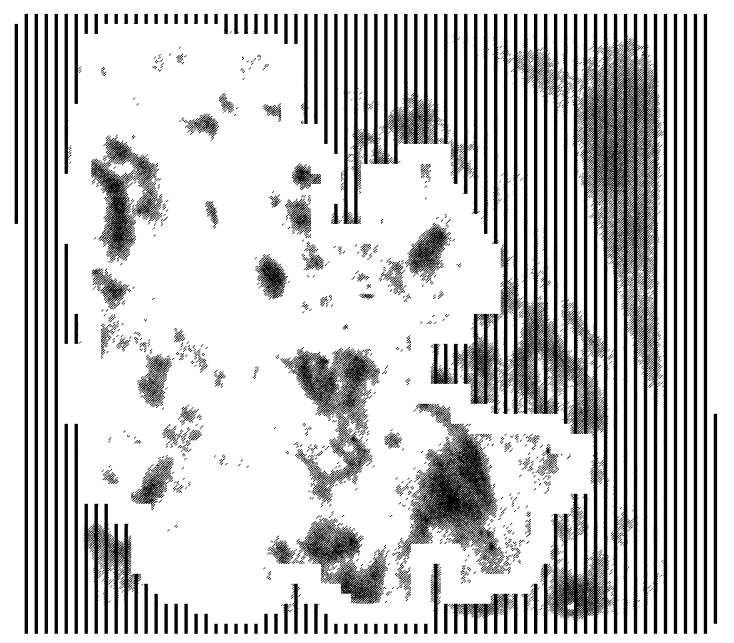

Fig. 8. A magnified observation of the same tumor.

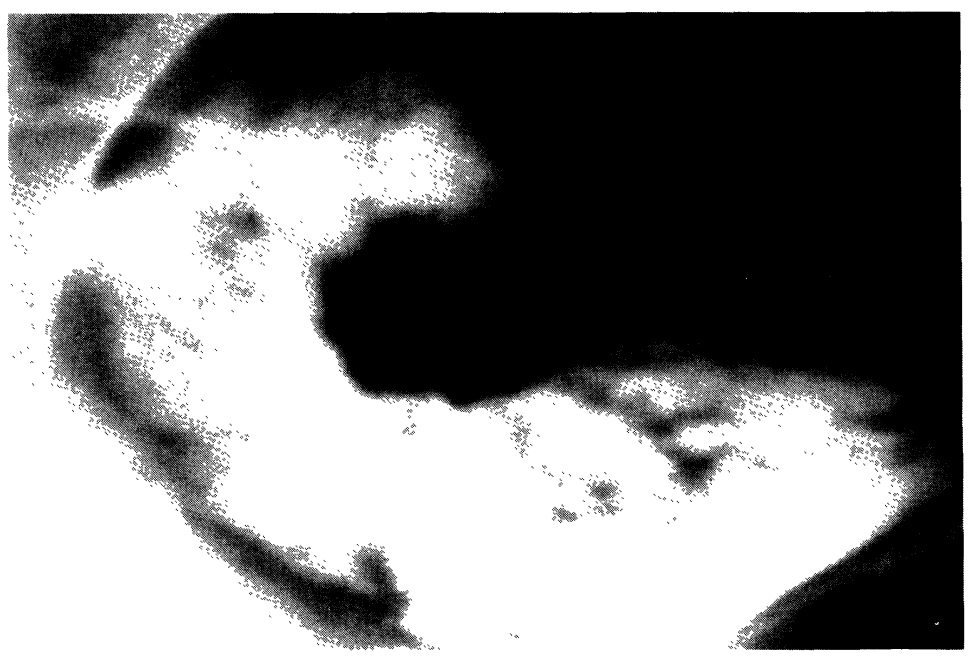

Fig. 9. The submucosographical frontal view showing a tumor as a vascular tumor from the relation of the mass with the surrounding blood vessels.

a mass survey and was suspected of having an abnormality on the gastric fundus. Gastrofiberscopy confirmed a semispherical reddish mass, with the mucosal feature of the surface suggestive of a gastric hemangioma (Fig. 7). Fig. 8 shows its magnified view. The submucosographic frontal view (Fig. 9) clearly visualized the relation of the mass with the surrounding blood vessels, establishing the diagnose of the mass as a vascular tumor. Pathohistologically, the tumor was a cavernous hemangioma. 


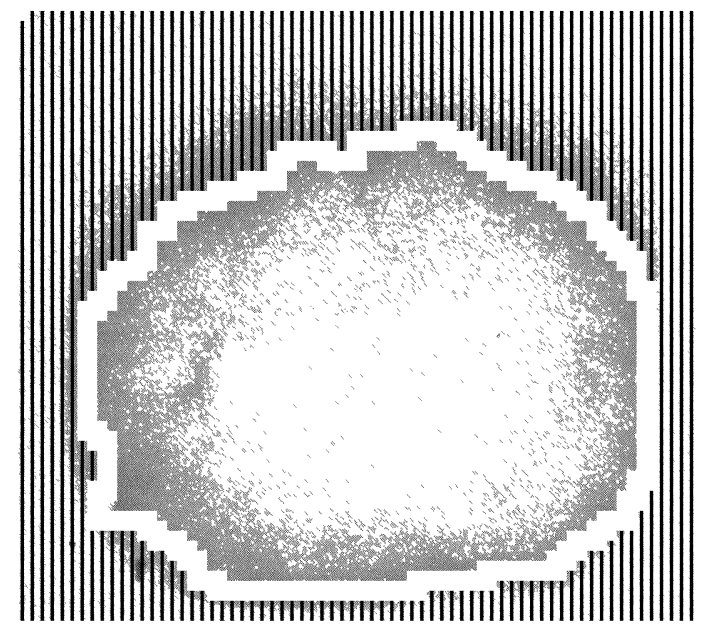

Fig. 10. Endoscopic picture showed a round submucosal tumor.

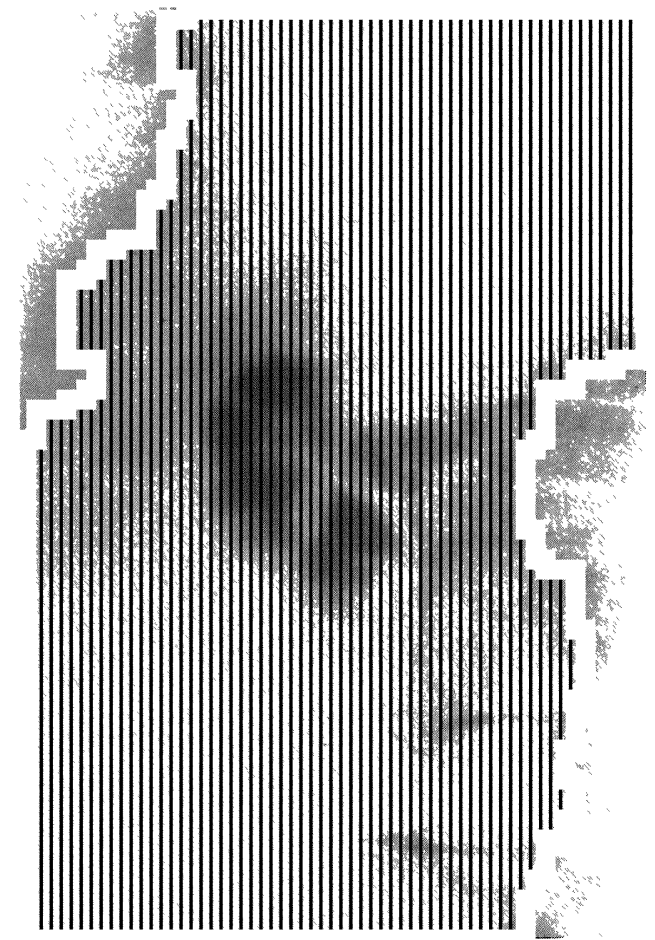

Fig. 11. Submucosography of the tumor shown in Fig. 10 revealed the lesion as a gastric varix with several submucosal veins. 


\section{Discussion}

There are gastric varices and hemangiomas that are sometimes indistinguishable from gastric cancer and other tumors. Indiscrete biopsy on such vascular tumors may cause serious bleeding, eventually requiring an emergency operation (Wakayama et al. 1977). By studying the relation between a submucosal mass and the surrounding blood vessels by the submucosography, we were able to identify an idiopathic gastric varix (Case 2), and to diagnose a mass as a vascular tumor (Case 3). To obtain histological specimens without danger or to perform endoscopic treatments, it is necessary to confirm whether the mass is a nonvascular tumor or not by some diagnostic procedure such as submucosography. Today, most abdominal masses can be easily diagnosed by CT scan or ultrasonography, but some of them can not, because they are too small or too intricate to exclude a bulging caused by an extragastric tumor. Application of submucosography can easily solve these problems. Case 1 in this paper was first recognized by both roentgenographical and endoscopical findings as having a mass on the anterior wall of the gastric fundus. Later by submucosography, the mass was confirmed as a tumescent lesion caused by compression of an extra-gastric mass at the left hepatic lobe. The patient was hospitalized for further detailed examinations. Hepatic ultrasonography, CT scan, and angiography all indicated a hemangioma in the left lobe of the liver. Further substantiated by peritoneoscopy, the mass was conclusively diagnosed as a hemangioma, and the patient underwent a surgical operation.

In clinical practice, idiopathic gastric varix or hemangioma is rare. However, in performing endoscopic biopsy to obtain histological specimens of a mass, attention should be paid to avoid incidental major bleeding. Injection of a contrast medium should be made at a point over $5 \mathrm{~mm}$ away from the lesion while the patient holds his breath to avoid injury by the injecting needle. In lesions suggesting a gastric varix, the application of submucosography demands the utmost discretion to avoid any serious fault. We experienced a round tumor-like lesion (Fig. 10). The injection of a water soluble contrast medium revealed that the lesion was a varix with several submucosal veins (Fig. 11). In this instance our precautionary submucosography eliminated the necessity of biopsy and thereby avoided the risk of an incidental major bleeding. Table 2 lists similar cases which were freed from unnecessary biopsy due to the submucosographic diagnosis of the lesion as a varix or hemangioma. In Case 1, the picture of a protruded mass endoscopically suggested a gastric submucosal tumor. However, our submucosography reasonably indicated it as a tumescent lesion caused by compression of an extra-gastric tumor. Accordingly an unnecessary endoscopic treatment was eliminated. If endoscopic resection using high frequency electric currents had been applied, a serious perforation might have been unavoidable. Up to the present there have been no measures to discriminate submucosal tumors 
from extra-gastric compression. And since angiographic diagnosis of the gastric submucosal tumor is still clinically difficult, submucosography as a non-invasive method is recommended.

\section{References}

1) Asaki, S., Hatori, S., Iwai, S., Nishimura, T., Sato, A. \& Goto, Y. (1982) Diagnosis of submucosal tumors by injecting a water soluble contrast medium. Basic research and imaging of tumors. Tohoku J. exp. Med., 138, 121-130.

2) Fujii, K., Yamagata, S., Suzuki, J., Sasaki, R., Shoji, T., Makabe, M., Memezawa, H. \& Maesawa, S. (1972) Angiographic features of submucosal tumors of the stomach. Tohoku J. exp. Med., 107, 287-299.

3) Nakayama, S., Matsuzaka, T., Koga, S., Kodama, K., So, M. \& Soejima, K. (1977) Cavernous hemangioma of the stomach, a case report. Rinshogeka, 32, 1329-1333. (Japanese) 